\title{
Evaluation of device reliability based on accelerated tests
}

\author{
E. Nogueira Díaz \\ Telefónica $I+D$, UPM, Spain \\ M. Vázquez López \& D. Rodríguez Cano \\ EUITT-Universidad Politécnica Madrid, Spain
}

\begin{abstract}
Reliability evaluation based on degradation is very useful in systems with scarce failures. In this paper a new degradation model based on Weibull distribution is proposed. The model is applied to the degradation of Light Emitting Diodes (LEDs) under different accelerated tests. The results of these tests are in agreement with the proposed model and reliability function is evaluated.
\end{abstract}

\section{INTRODUCTION}

Reliability evaluation based on degradation models[1] is commonly applied in highly reliable products as a cost effective and confident way of evaluating their reliability. In this paper a devices degradation model is presented and subsequently applied in the quantitative analysis of LEDs reliability. With this model the different parameters related to module reliability, such as the reliability function, failure rate function, the Mean Time to Failure (MTTF) or the warranty period can be assessed based on LED degradation. In order to obtain reliability data in a suitable period of time degradation is measured in climatic chamber in accelerated tests.

The classical degradation model determines the number of failures at any time based on degradation data. This model assumes that functionality parameter, light output in the case of LEDs, of a group of devices follows a normal distribution in each instant of time, whose parameters (mean and standard deviation) change as a function of time.

In this paper the classical model limitations, from theoretical and practical point of view, are analysed. The calculations were performed in order to see the temporal limitation of the classic model, using mean and standard deviation linear variation with time. The standard deviation trend limits are also analysed in order to avoid non real results from the degradation point of view as LEDS that improve with time and light output values lower than zero.

Finally, we propose a model using the Weibull distribution to solve the classical model limitations.

\section{CLASSIC MODEL}

In degradation models it is assumed that a component fails when one of its functional parameters (power, voltage, light output etc) degrades enough that does not allow it to carry out its functionality successfully. Degradation failure is usually defined as a percentage of the nominal value from which the component is considered to be unable to perform its function. For example in LEDs case, the failure is considered when the light output falls below $70 \%$ of nominal value [2].

Classical model assumes that:

- The functionality parameter is distributed following a normal distribution with an average, $\mu$, and standard deviation, $\sigma$.

- Average and standard deviation are functions of time, $\mu(\mathrm{t})$ and $\sigma(\mathrm{t})$.

For the mean a linear variation is usually used by several authors [3-4].

$\mu(\mathrm{t})=\mu_{0}-\mathrm{At}$

Where:

$\mu_{0}$ mean initial value

A constant that indicates the speed of degradation. $\mathrm{t}$ time.

Linear trend presents a problem for $t \geq \mu_{0} / \mathrm{A}$ because, in this period of time, functionality parameter takes values lower than zero. 
Other authors [5] propose an exponential trend:

$\mu(\mathrm{t})=\mu_{0} \mathrm{e}^{-\mathrm{t} / \mathrm{C}}$

being:

$\mu_{0}$ mean initial value

C Constant that represents the time for which the parameter has degraded to a $36.7 \%$ of its initial value.

For the time variation of the standard deviation is often used a linear variation:

$\sigma(\mathrm{t})=\sigma_{0}+\mathrm{B} \mathrm{t}$

Where:

$\sigma_{0}$ initial standard deviation.

$\mathrm{B}$ constant that indicates the speed of degradation of standard deviation.

$\mathrm{t}$ time.

In general this model assumes that the parameter distribution in any instant of time follows a normal distribution with an average $\mu(t)$ and standard deviation $\sigma(\mathrm{t})$.

$f(p, t)=\frac{1}{\sigma(t) \sqrt{2 \pi}} e^{-\frac{1}{2}\left(\frac{p-\mu(t)}{\sigma(t)}\right)^{2}}$

Figure 1 shows the previous model assuming a linear variation of both the average and the standard deviation.

Due the standard deviation increases with time, the normal distribution will be flatting with time, and therefore in a certain instant of time the normal curve,

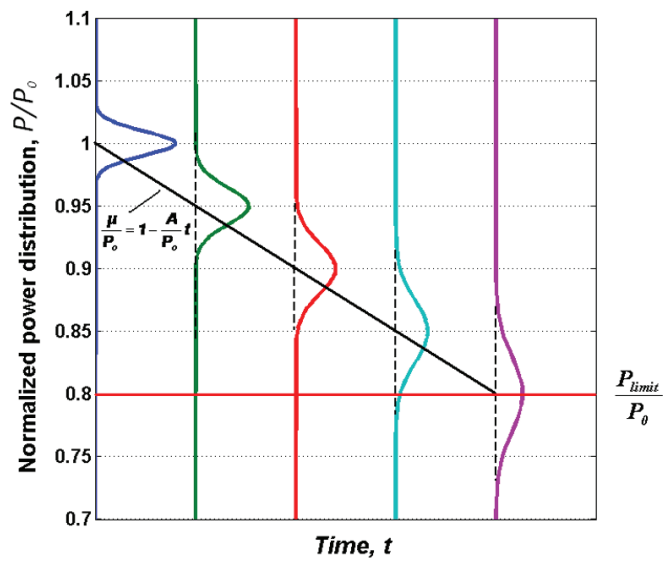

Figure 1. Mean and standard deviation evolution with time. or in a simpler way $\mu(t)-3 \sigma(t)$, will pass through the failure limit, and in that moment degradation failures will appear.

Based on this model it is possible to evaluate the reliability as the probability in any instant of time that functionality parameter is within the non-failure parameter limits.

$R(t)=\int_{L_{L}}^{L_{S}} \frac{1}{\sigma(t) \sqrt{2 \pi}} e^{-\frac{1}{2}\left(\frac{p-\mu(t)}{\sigma(t)}\right)^{2}} d p$

Where:

$\mathrm{p}$ Parameter that is being analysed

$\mu$ mean

$\sigma$ standard deviation

$\mathrm{L}_{\mathrm{L}}$ and $\mathrm{L}_{\mathrm{S}}$ are the lower and upper failures limits.

There are some device manufacturers that provide degradation data but they are scarce. In order to obtain data from degradation in a suitable period of time it is necessary to use accelerate tests as it will be explained in this paper.

Reliability from degradation data can be estimated using the equation (5). One time parameter that is easily evaluated with this model following the Figure 1 is the time at which $50 \%$ of the devices failed $\mathrm{R}\left(\mathrm{t}_{50}\right)=0.5$.

In the linear parameter trend case $t_{50}$ will be:

$t_{50}=\frac{\mu_{0}-p_{F}}{A}$

$\mathrm{p}_{\mathrm{F}}$ failure limit parameter.

In the exponential parameter trend case $t_{50}$ will be:

$t_{50}=-C \ln \frac{p_{F}}{\mu_{0}}$

It is also easily evaluated the time at which reliability is practically zero. This time can be calculated by means of the following equation:

$\mu(\mathrm{t})=\mathrm{p}_{\mathrm{F}}-3 \sigma(\mathrm{t})$

\section{CLASSIC MODEL LIMITATIONS}

Using classic model, and depending on the degradation parameter, it is possible to obtain results without any physical sense. As an example, it is possible that using the classic model a percentage of the devices improves their performance, functionality parameter, $\mathrm{s}$ it can be seen in Figure 2.

As can be seen in Figure 2 there is a percentage of devices (calculated as $\mu(t)+3 \sigma(t)$ ) that improves their performance with time that is not possible in a 


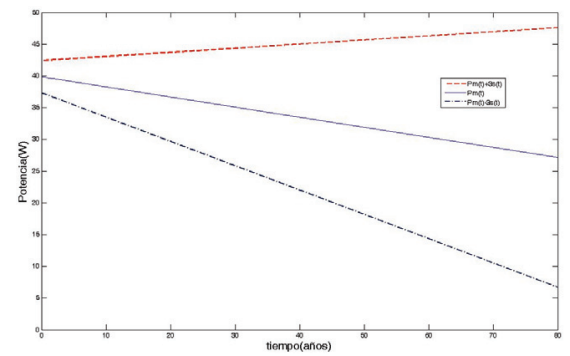

Figure 2. Normal distribution power values $(\mu+\sigma, \mu, \mu-\sigma)$ with mean and standard deviation linear trend according to the classical model.

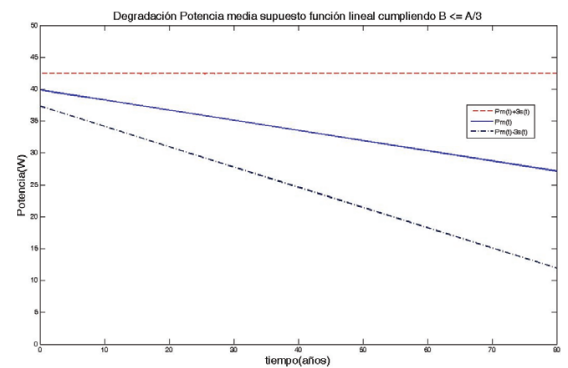

Figure 3. Normal distribution power values $(\mu+\sigma, \mu, \mu-\sigma)$ with mean and standard deviation linear trend according to the classical mode $(\mathrm{A} \approx 3 \mathrm{~B})$.

degradation model. In order to avoid this situation it is necessary that degradation trend follows the next equation:

$\mu(t)+3 \sigma(t) \leq \mu_{0}+3 \sigma_{0}$

In the case of mean and standard linear trend it is necessary that:

$$
3 \mathrm{~B} \geq \mathrm{A}
$$

In the next figure it can be seen a case in which $\mathrm{A} \approx 3 \mathrm{~B}$

In the exponential average degradation case the analysis is very similar to the previous case as it can be seen in Figure 4.

\section{PROPOSED MODEL}

In the paper we propose a model that is based on the assumptions that functionality parameter decays with time following a Weibull distribution function[6]. Weibull function is very useful due its versatility

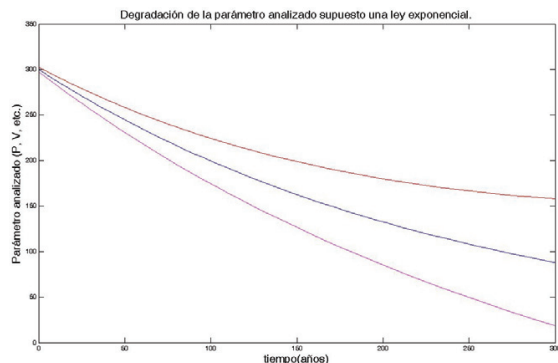

Figure 4. Normal distribution power values $(\mu+\sigma, \mu, \mu-\sigma)$ with average exponential trend and standard deviation linear trend according to the classical model.

because depending on the parameters is possible to approximate very different degradation trends.

In Weibull distribution functionality parameter depends on time in the following way:

$\mu(t)=\mu_{0} e^{-\left(\frac{t-t_{0}}{\eta}\right)^{\beta}}$

being

$\mathrm{t}_{0}$-location parameter.

$\eta$-scale parameter.

$\beta$-shape parameter (or slope).

Related scale parameter:

If $\beta=1$ functionality parameter varies with respect time following an exponential. It means that degradation rate is constant in the whole period of time:

$\frac{\eta(t+\Delta t)}{\eta(t)}=\frac{\eta_{0} e^{-\left(\frac{t+\Delta t}{\eta}\right)}}{\eta_{0} e^{-\left(\frac{t}{\eta}\right)}}=e^{-\Delta t}$

If $\beta<1$ degradation rate decreases with time.

If $\beta>1$ degradation rate increases with time.

$\eta$ is the scale parameter and is the time at which the functionality parameter has reduced to $\mathrm{e}^{-1}(0.368)$. $\mathrm{t}_{0}$ - location parameter, defines the degradation starting point.

For the common case where $t_{0}=0$ and $\eta=1$ the figure shows three curves for the three types of $\beta$ described in the preceding paragraph.

Main advantages of Weibull function are:

- It takes values between $\mu_{0}$ in $\mathrm{t}=0$ and $0(\mathrm{t}=$ infinite) according theoretical degradation models. Although takes zero value for $t$ equal to infinite it is possible to model practical zero value at finite times. 


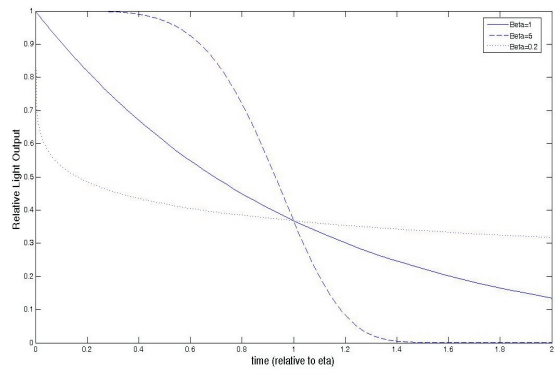

Figure 5. Weibull distribution for $t_{0}=0, \eta=1$ and different $\beta$ values $(0.2,1$ and 5).

- Different parameters degradations reported in the literature as linear degradation, exponential or others can be modeled with a Weibull distribution.

- Weibull parameters give us information about the degradation rate (constant, decreases with time or increases with time).

\section{EXPERIMENTAL TESTS}

Degradation model has been applied to experimental results obtained in LEDs that has been working in a Pressure Cooker chamber under different temperature and humidity conditions. LEDs were working during the tests in the pressure cooker chamber according to the next figure.

Test procedure was:

1. 15 LEDs were introduced in the Presure Cooker applying a bias volage according to the scheme shown in the figure. Polarization resistance and power supply were outside of the camera.

2. Periodically, once each day, the test was interrupted and all the LEDs were characterized with an optical power meter.

3. Luminosity power has been represented and compared with initial power luminosity.

In the $110^{\circ} \mathrm{C} / 85 \%$ case the test lasted 29 days and in the 19 day began to appear catastrophic failures. Catastrophic failures have not been taken into account in calculating means and standard deviations.

\section{DEGRADATION MODEL VERIFICATION}

In order to analyse the model validity we have evaluated different subjects:

1. Power luminosity of the 15 LEDs follows a normal distribution in any instant of time. Average and standard deviation have been calculated by means of normal representation.

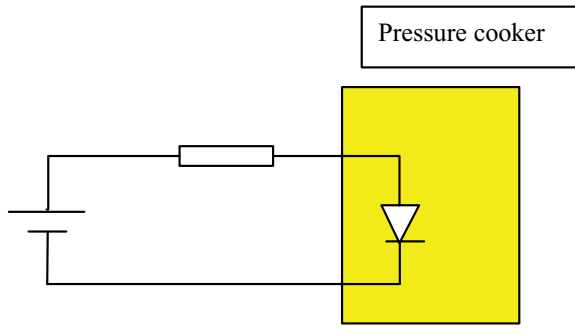

Figure 6. Test circuit.

Table 1. Power luminosity values for the days 10,17 and 26. Pressure Cooker $110^{\circ} \mathrm{C} / 85 \% \mathrm{RH}$

\begin{tabular}{llll}
\hline & \multicolumn{2}{l}{ Day } & \\
\cline { 2 - 4 } LED & 10 & 17 & 26 \\
\hline L1 & 0,552 & 0,322 & 0,183 \\
L2 & 0,574 & 0,488 & 0,316 \\
L3 & 0,613 & 0,549 & 0,476 \\
L4 & 0,591 & 0,538 & 0,294 \\
L5 & 0,616 & 0,560 & 0,534 \\
L6 & 0,606 & 0,524 & 0,400 \\
L7 & 0,634 & 0,384 & 0,265 \\
L8 & 0,606 & 0,623 & 0,551 \\
L9 & 0,610 & 0,567 & 0,481 \\
L10 & 0,623 & 0,385 & 0,171 \\
L11 & 0,604 & 0,489 & 0,000 \\
L12 & 0,628 & 0,570 & 0,462 \\
L13 & 0,629 & 0,600 & 0,521 \\
L14 & 0,575 & 0,519 & 0,000 \\
L15 & 0,565 & 0,535 & 0,410 \\
\hline
\end{tabular}

2. Power luminosity degrades following a Weibull function. Weibull representation has been used for this purpose.

\subsection{LEDs Power luminosity distribution}

We have Table 1 shows the 15 LEDs power luminosity results for the days ten, seventeen and twenty-six in the test $110^{\circ} \mathrm{C} / 85 \% \mathrm{RH}$ in the Pressure Cooker.

As can be seen in Table 1 on day 26, two LEDs, 11 and 14 , have failed catastrophically. These Power luminosity data have bee represented in a normal paper as it can be seen in Figure 7. From these normal representations average and standard deviation from different instant of time have been evaluated.

From normal plot average and standard deviation values are easily evaluated. In the following figures the average and standard deviation power luminosity with respect time have been represented. 


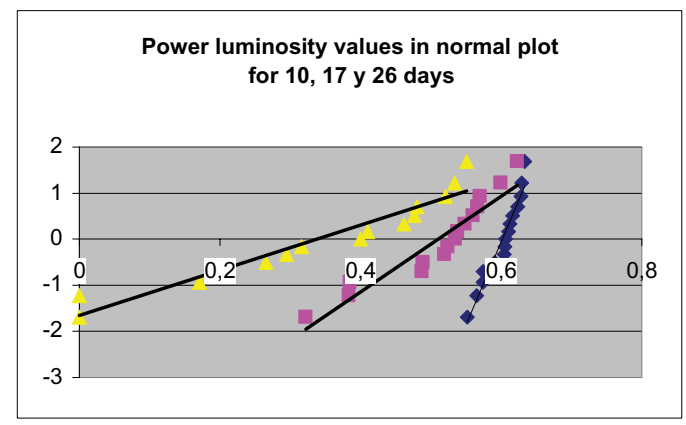

Figure 7. Normal distribution representation in three different instant of time (day 10-blue and right, day 17pink-middle and day 26-yellow-left). Pressure cooker test $\left(110^{\circ} \mathrm{C} / 85 \% \mathrm{RH}\right)$

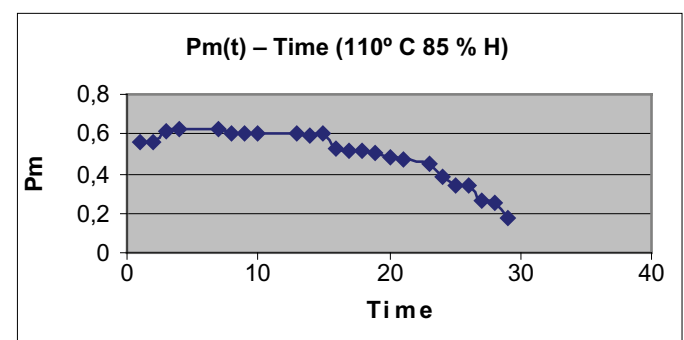

Figure 8. Average Power luminosity respect time.

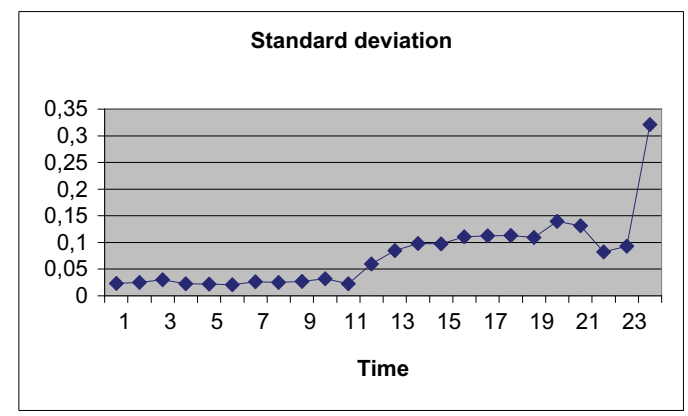

Figure 9. Standard deviation Power luminosity respect time.

From Figure 8 it can be seen the behaviour of power luminosity versus time. During the first days power luminosity increases with respect initial power luminosity and after that the device starts to degrade. This evolution has been reported in the literature by other authors $[7,8]$.

From standard deviation evolution it is possible to distinguish three different periods: the first period is

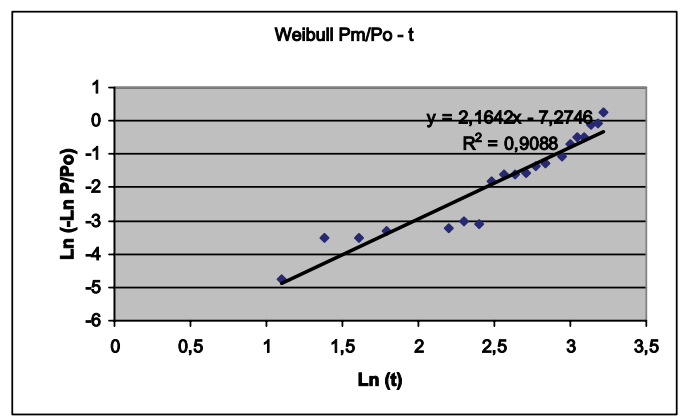

Figure 10. Power luminosity vs time in a Weibull plot.

from the beginning till eleven day and the standard deviation is almost constant. Second period is from eleven to eighteen day and in this period the standard deviation increases following a linear trend, according to the classical model. In the last period that starts in 19 day catastrophic failures appear and therefore it is not easy to find the standard deviation trend.

\subsection{Power luminosity Weibull function}

Based on the figure 7 we have evaluated average power luminosity with respect time. It can be seen that degradation does not start until day 4 . In figure 10 we have represented the relative power luminosity (relative to power luminosity at fourth day) with respect time in a Weibull representation concluding that can be modelled with a Weibull function.

From Weibull representation we have obtained Weibull parameters for this specific test $\left(110^{\circ}\right.$ $\mathrm{C} / 85 \% \mathrm{RH})$.

As can be seen following the proposed law with $\beta=2.1642, \eta=28.8276$ days $=691,86$ hours, and therefore power luminosity evolutions with time in the following way.

$P_{m}(t)=0,62 e^{-\left(\frac{t-96}{691,86}\right)^{2.16}}$.

\section{RELIABILITY EVALUATION}

We have evaluated reliability function and MTTF assuming that device failure limit is $70 \%$ of nominal luminosity. Table 2 shows in which days the failures appear.

We have represented the failures in Weibull plot as it can be seen in Figure 8.

From Weibull plot it is possible to evaluate reliability function obtaining a $\beta$ value higher than one for all the tests indicating a degradation mechanism. 
Table 2. Accumulated

failures at different days

$\left(110^{\circ} \mathrm{C} / 85 \% \mathrm{RH}\right)$.

\begin{tabular}{ll}
\hline Days & Accumulated failures \\
\hline 12 & 1 \\
13 & 3 \\
16 & 4 \\
20 & 8 \\
22 & 9 \\
23 & 11 \\
24 & 14 \\
25 & 15 \\
\hline
\end{tabular}

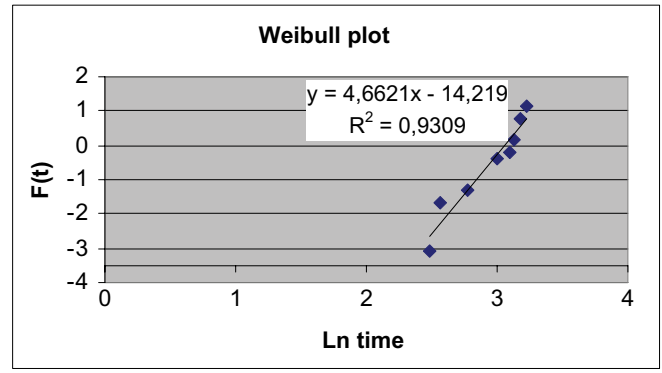

Figure 11. Weibull plot for $110^{\circ} \mathrm{C} / 85 \% \mathrm{RH}$ pressure cooker test.

\section{RESULTS AND FUTURE WORK}

Once average and standard deviation power luminosity has been evaluated it is easy to evaluate reliability based on the proposed model. First results show that:

- All the experiments done at different conditions $\left(110^{\circ} \mathrm{C} / 85 \% \mathrm{RH}, \quad 130^{\circ} \mathrm{C} / 85 \% \mathrm{RH}\right.$ and $\left.140^{\circ} \mathrm{C} / 85 \mathrm{RH}\right)$ inside the pressure cooker chamber can be fitted by the proposed model.

- First results related with reliability evaluation by the proposed model show that all of them have similar degradation behaviour but at different rates depending on the accelerated tests.

- After several hours of operation, this time depends on the acceleration factor, LEDs start degradation and failure rate increases with time.

- Tests at different temperature/humidity conditions are on going in order to analyse the reliability influence of the different parameters: temperature, humidity and pressure.

- Reliability at normal working conditions will be evaluated when all the tests will be finished.

\section{CONCLUSIONS}

Main conclusions of this paper are:

- We have proposed a degradation model based on Weibull function that fits with several degradation models for different devices reported in the literature.

- From this model it is possible to evaluate the reliability function of any device by means of analyzing degradation data.

- First results in accelerated tests in AlGaInP LEDs shows that:

- LEDs degradation follows a Weibull function respect time in agreement with the proposed model.

- Reliability in all pressure cooker tests follows a Weibull function with a shape parameter higher than one in agreement with the degradation mechanism.

- Accelerated tests at different conditions are on going in order to extrapolate reliability data at normal working conditions.

\section{REFERENCES}

[1] Coit D.W., Evans J.L., Vogt N.T., Thomson J.R. A method for correlating field life degradation with reliability prediction for electronic modules. Quality and Reliability Engineering International 2005; 21: 715-726.

[2] ASSIST: Alliance for Solid-State Illumination Systems and Technologies. LED life for general lighting. Assist Recommends 2005; 1(1):1-13.

[3] Osterwald C.R., Benner J.P., Pruett J., Anderberg A., Rummeland S., Ottoson L. Degradation in weathered crystalline-silicon PV modules apparently caused by UV radiation. 3rd World Conference on Photovoltaic Energy Conversion.

[4] Jia-Sheng Huang. "Reliability-Extrapolation Methodology of Semiconductor Laser Diodes." IEEE Transactions on Device and Materials Reliability, Vol. 6, NO 1, (2006).

[5] Xie J., Pecht M. "Reliability Predicition Modeling of Semiconductor Light Emitting Device.” IEEE Transactions on Device and Materials Reliability Vol 6, No3. 218-222.

[6] Weibull W. "Fatigue Testing and Analysis of results" Pergamon Press New York.

[7] Kish F.A., Vanderwater D.A., DeFevere D.C., Steigerwald D.A., Hofler G.E., Park K.G., Steranka F.M. "Highly reliable and efficient semiconductor waferbonded AlGaInP/GaP light-emitting diode." Electronics Letters Vol. 32, No. 2; 132-134 (1996).

[8] Grillot P.N., Krames M.R., Zhao H., Teoh S.H. "Sixty Thousand Hour Light Output Reliability of AlGaInP Light Emitting Diodes." IEEE Transactions on Device and Materials Reliability. Vol. 6, No. 4; 564-574 (2006). 